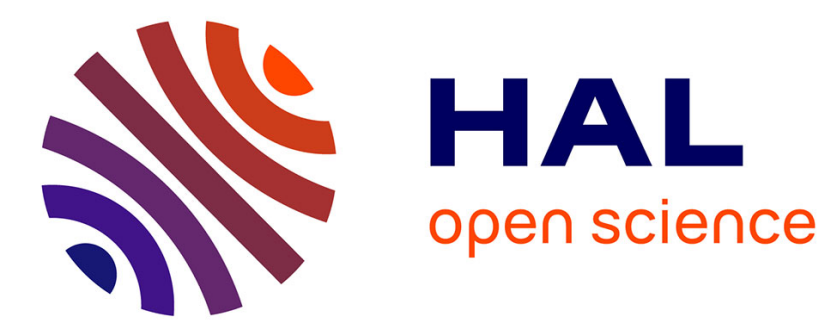

\title{
Associations between cognitive restraint, history of weight loss diet and organic food consumption in a large population-based sample of adults
}

Sandrine Péneau, Kelly Virecoulon-Guidici, Julia Baudry, Caroline Méjean, Denis Lairon, Marc Bénard, Serge Hercberg, Emmanuelle Kesse-Guyot

\section{To cite this version:}

Sandrine Péneau, Kelly Virecoulon-Guidici, Julia Baudry, Caroline Méjean, Denis Lairon, et al.. Associations between cognitive restraint, history of weight loss diet and organic food consumption in a large population-based sample of adults. 42. Anniversary Meeting of the British Feeding and Drinking Group (BFDG), Apr 2018, Ecullly-Lyon, France. 1 p., 2018. hal-02786241

\section{HAL Id: hal-02786241 \\ https://hal.inrae.fr/hal-02786241}

Submitted on 4 Jun 2020

HAL is a multi-disciplinary open access archive for the deposit and dissemination of scientific research documents, whether they are published or not. The documents may come from teaching and research institutions in France or abroad, or from public or private research centers.
L'archive ouverte pluridisciplinaire HAL, est destinée au dépôt et à la diffusion de documents scientifiques de niveau recherche, publiés ou non, émanant des établissements d'enseignement et de recherche français ou étrangers, des laboratoires publics ou privés. 


\title{
Associations between cognitive restraint, history of weight loss diet and organic food consumption in a large population-based sample of adults
}

\author{
Sandrine Péneau, Kelly Virecoulon Giudici ${ }^{1}$, Julia Baudry ${ }^{1}$, Caroline Méjeann ${ }^{1,2}$, Denis Lairon ${ }^{3}$, Marc Bénard ${ }^{1}$, \\ Serge Hercberg ${ }^{1,4}$, Emmanuelle Kesse-Guyot ${ }^{1}$
}

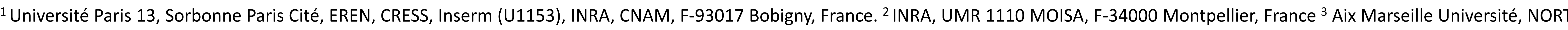
; Insem, UMR S 1062 ; Inra 1260, 13385 Marseille, France. ${ }^{4}$ Université Paris 13, Sorbonne Paris Cité, USEN, F-93017, Bobigny, France; InVS, F-93017, Bobigny, France.

\section{Background}

- Organic food consumption has risen in many countries during the past decades, but the links with psychological factors and dieting remain unclear.

- The aim of this study was to evaluate the associations between cognitive restraint, weight loss diet history and organic food intake, in French adults.

\section{Methods}

- Participants of the NutriNet-Santé study, a large ongoing web-based prospective cohort started in France in May 2009.

- 21,516 participants (5,688 men, 15,828 women) in this cross-sectional study

- Cognitive restraint : evaluated by the Three-Factor Eating Questionnaire

- Practice of weight-loss diet in the past years : assessed by an ad hoc questionnaire

- Organic food intake of 17 food groups : assessed by the Organic-Food Frequency Questionnaire.

- Logistic regression models were performed and proportions of organic food intakes out of total food intakes were compared.

- Analyses were adjusted for age, education, occupation, income, urban unit size, family situation, energy intake, total intake of the group, physical activity level, mPNNS-GS, BMI

\section{Results}

\section{- Cognitive restraint}

Table 1: Multivariable logistic regression analysis showing the association between quartiles of cognitive restraint score and organic food intake in women

\begin{tabular}{|c|c|c|c|c|c|}
\hline & Q1 & Q2 & Q3 & Q4 & $p^{1}$ \\
\hline Fruit and vegetables & Ref & $\begin{array}{c}0.98 \\
(0.86-1.11)\end{array}$ & $\begin{array}{c}0.96 \\
(0.84-1.10)\end{array}$ & $\begin{array}{c}0.87 \\
(0.76-0.99)\end{array}$ & 0.23 \\
\hline Fish & Ref & $\begin{array}{c}0.99 \\
(0.90-1.08)\end{array}$ & $\begin{array}{c}1.02 \\
(0.93-1.12)\end{array}$ & $\begin{array}{c}0.96 \\
(0.87-1.05)\end{array}$ & 1.00 \\
\hline $\begin{array}{l}\text { Red meat, poultry, processed } \\
\text { meat }\end{array}$ & Ref & $\begin{array}{c}0.95 \\
(0.85-1.05)\end{array}$ & $\begin{array}{c}0.96 \\
(0.86-1.08)\end{array}$ & $\begin{array}{c}0.87 \\
(0.78-0.97)\end{array}$ & 0.07 \\
\hline Dairy products & Ref & $\begin{array}{c}0.99 \\
(0.90-1.09)\end{array}$ & $\begin{array}{c}0.90 \\
(0.81-0.99)\end{array}$ & $\begin{array}{c}0.97 \\
(0.88-1.07)\end{array}$ & 0.33 \\
\hline Starchy refined foods & Ref & $\begin{array}{c}0.99 \\
(0.89-1.10)\end{array}$ & $\begin{array}{c}0.93 \\
(0.83-1.04)\end{array}$ & $\begin{array}{c}0.93 \\
(0.84-1.04)\end{array}$ & 0.78 \\
\hline Fast food & Ref & $\begin{array}{c}0.99 \\
(0.91-1.09)\end{array}$ & $\begin{array}{c}0.93 \\
(0.85-1.03)\end{array}$ & $\begin{array}{c}0.89 \\
(0.81-0.98)\end{array}$ & 0.13 \\
\hline Fatty sweets & Ref & $\begin{array}{c}1.00 \\
(0.90-1.11)\end{array}$ & $\begin{array}{c}0.86 \\
(0.77-0.96)\end{array}$ & $\begin{array}{c}0.88 \\
(0.79-0.98)\end{array}$ & 0.021 \\
\hline Non-fatty sweets & Ref & $\begin{array}{c}0.97 \\
(0.87-1.07)\end{array}$ & $\begin{array}{c}0.88 \\
(0.79-0.97)\end{array}$ & $\begin{array}{c}0.87 \\
(0.78-0.96)\end{array}$ & 0.036 \\
\hline Fats & Ref & $\begin{array}{c}0.92 \\
(0.83-1.02)\end{array}$ & $\begin{array}{c}0.85 \\
(0.77-0.95)\end{array}$ & $\begin{array}{c}0.87 \\
(0.78-0.96)\end{array}$ & 0.031 \\
\hline Non-alcoholic drinks & Ref & $\begin{array}{c}0.91 \\
(0.83-1.01)\end{array}$ & $\begin{array}{c}0.85 \\
(0.77-0.95)\end{array}$ & $\begin{array}{c}0.82 \\
(0.74-0.91)\end{array}$ & 0.004 \\
\hline Alcoholic beverages & Ref & $\begin{array}{c}0.96 \\
(0.87-1.05)\end{array}$ & $\begin{array}{c}0.98 \\
(0.89-1.09)\end{array}$ & $\begin{array}{c}0.87 \\
(0.79-0.96)\end{array}$ & 0.07 \\
\hline
\end{tabular}

In addition

Women with higher levels of cognitive restraint presented lower mean \% of organic food (all food groups), compared with those with lower level of cognitive restraint

Only a few association were observed in men

\section{- History of weight loss diet}

Table 2: Multivariable logistic regression analysis showing the association between history of weight loss diet and organic food intake in women

\begin{tabular}{|c|c|c|c|}
\hline & Never dieters & Dieters (past/current) & $\mathrm{p}^{1}$ \\
\hline Fruit and vegetables & Ref & $\begin{array}{c}0.95 \\
(0.86-1.05)\end{array}$ & 0.60 \\
\hline Fish & Ref & $\begin{array}{c}1.03 \\
(0.96-1.10)\end{array}$ & 0.88 \\
\hline $\begin{array}{l}\text { Red meat, poultry, processed } \\
\text { meat }\end{array}$ & Ref & $\begin{array}{c}1.00 \\
(0.92-1.08)\end{array}$ & 0.92 \\
\hline Dairy products & Ref & $\begin{array}{c}0.99 \\
(0.92-1.07)\end{array}$ & 1.00 \\
\hline Starchy refined foods & Ref & $\begin{array}{c}1.02 \\
(0.94-1.11)\end{array}$ & 1.00 \\
\hline Fast food & Ref & $\begin{array}{c}0.89 \\
(0.82-0.95)\end{array}$ & 0.004 \\
\hline Fatty sweets & Ref & $\begin{array}{c}0.93 \\
(0.86-1.01)\end{array}$ & 0.20 \\
\hline Non-fatty sweets & Ref & $\begin{array}{c}0.91 \\
(0.84-0.98)\end{array}$ & 0.049 \\
\hline Fats & Ref & $\begin{array}{c}0.94 \\
(0.87-1.02)\end{array}$ & 0.31 \\
\hline Non-alcoholic drinks & Ref & $\begin{array}{c}0.91 \\
(0.84-0.98)\end{array}$ & 0.037 \\
\hline Alcoholic beverages & Ref & $\begin{array}{c}0.90 \\
(0.83-0.97)\end{array}$ & 0.017 \\
\hline
\end{tabular}

\section{In addition}

Women who were past or current dieters presented lower mean $\%$ of organic fast food, fatty sweets, non fatty sweets, fats, non-alcoholic and alcoholic beverages compared with non dieters

No association was observed in men 